\title{
Evaluation of Vickers-Trexler isolator in children undergoing bone marrow transplantation
}

\author{
J. G. WATSON, T. R. ROGERS, S. SELWYN, AND R. G. SMITH
}

From Westminster Children's Hospital and Department of Bacteriology, Westminster Medical School, London

SUMMARY Four children, 5 months to 15 years of age, underwent bone marrow transplantation in Vickers-Trexler isolator tents. Two grafts were elective. During 170 days of isolation no clinical infections due to exogenous micro-organisms developed despite severe immunodeficiency. The decontamination regimen and sterile procedures used, as well as the microbiological results, are described. This form of isolation in paediatric practice was found to be highly acceptable to both patients and staff.

Isolators have been used for many years to maintain 'germ-free' or gnotobiotic animal colonies (Gordon and Pesti, 1971) and more recently in the protection of adult leukaemia patients undergoing intensive treatment (Trexler et al., 1975). They have also been of value in the provision of a sterile environment for newborn babies with suspected immunological deficiency. We have used isolators for bone marrow transplantation in 4 children who were extremely susceptible to infection either due to the nature of their disease or as a result of pregraft immunosuppression. Two children had severe combined immune deficiency disease (SCID), one had chronic granulomatous disease (CGD), and one had Fanconi's hypoplastic anaemia. The marrow grafting procedures used have been described previously (Humble and Barrett, 1975).

The susceptibility to infection in these conditions depends on the degree to which cellular and humoral immunity are impaired. For example, polymorphonuclear leucocytes and monocytes in patients with CGD have greatly impaired ability to kill Staphylococcus aureus, Serratia, Aerobacter, and Candida species, and thus predispose to infection with these organisms (Oh et al., 1969; Rodey et al., 1969). Previous therapy and the duration of the disease or its complications will also markedly influence the bacterial flora present. Even after marrow transplantation there is an increased susceptibility to infection during the first 3 weeks due to granulocytopenia and for at least the first 3 months because of immunoincompetence (Johnson et al., 1976).

Received 28 November 1976

\section{Isolation and decontamination procedures}

In association with Mr. P. C. Trexler of the Royal Veterinary College (University of London) we have adapted isolators for paediatric use. The basic structure and management of the bed isolator (Fig. 1), including the supply of positive pressure sterile air using a $1415 \mathrm{l} / \mathrm{min}$ blower and high efficiency particle arrester (HEPA) filters, is very much as described previously (Trexler et al., 1975). The infant isolator (Fig. 2) is made of the same transparent polyvinyl chloride $(0 \cdot 1-0 \cdot 2 \mathrm{~mm}$ thickness). It consists of a sausage, $137 \times 91 \mathrm{~cm}$, which has four sleeves down each side-instead of half suits-and additional facilities for weighing and taking $x$-rays. The storage isolator and its manner of connection to both infant and bed versions are very similar. The infant isolator can be stored complete and sterile and is ready for use within 24 hours. The larger version is ready for use in $\mathbf{4 8}$ hours. Both isolators can be erected over standard hospital cots or beds in a normal ward and removed for storage when not required.

The pregraft decontamination regimens adopted were similar in all cases. The patients were initially nursed under reverse barrier conditions in a cubicle. All persons entering the cubicle wore hats, masks, gowns, gloves, and overshoes. All laundry was sterile. Good quality prepacked food was used and samples tested were found to be sterile on all occasions. All surfaces in the cubicle, including equipment, were disinfected with $1.5 \%$ Hycolin in spirit. The patients had daily baths after applying $4 \%$ chlorhexidine in detergent (Hibiscrub) to the skin; they also received thrice daily sprays of chlor- 


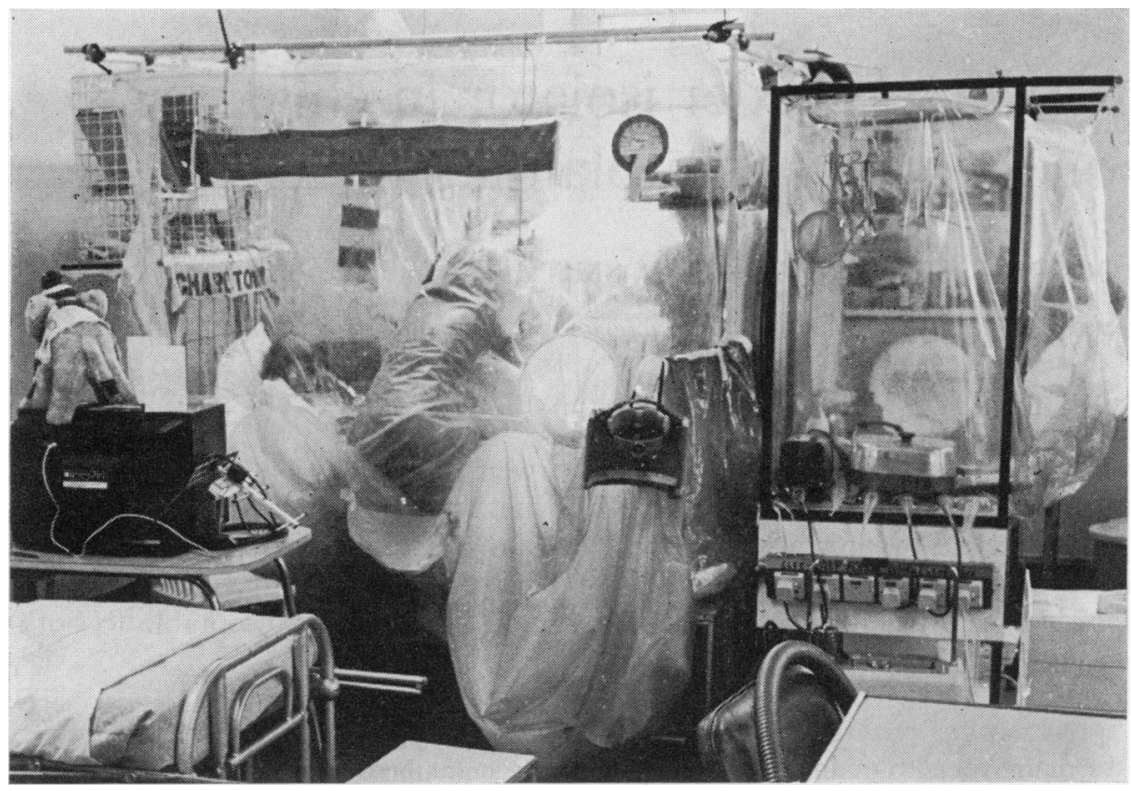

Fig. 1 Large isolator seen in a normal paediatric ward.

hexidine $(0.02 \%$ aqueous solution) to the nose, throat, ears, and foreskin and, on alternate days, the hair was washed with Savlon. Chlorhexidine obstetric cream was applied nightly to the vagina. Corsodyl (chlorhexidine) dental gel was applied to the gums and Naseptin to the nose twice daily.

Bowel decontamination in the nonelective cases was started in the cubicle, while in the elective cases it began in the isolator. Each patient received a combination of oral antibiotics according to the 'Fracon' regimen devised by Trexler et al. (1975). Administration of nystatin was started 4 days before framycetin and colistin and the doses of all antibiotics were modified according to age. Two items were changed due to patient preference. As a result of our experience with previous cases, Case 2 (Table) received proportionately lower doses of colistin to lessen the risk of gastric intolerance. The full decontamination regimen was continued until 72 hours before the planned removal of the patient from the isolator.

Once the microbial population at the various sites had reached minimal levels, the patient entered the isolator wearing only a sterile gown, which was shed on entry. Decontamination of the total body surface, bowel, and orifices was continued using sterile solutions and sterile drugs. All articles entering the isolator were sterilized as appropriate, by heat, gamma irradiation, or chemical means (Milton hypochlorite 1 in 80 ). Food was sterilized by gamma irradiation in commerically prepared packs. Samples for bacteriological culture were taken thrice weekly from nose, throat, mouth, urine, and vagina. Axillae, groins, hairline, ears, and any skin spots were sampled twice-weekly using swabs moistened with water for dry areas. Swabs from the tent were examined regularly. All specimens were cultured aerobically and anaerobically. The delay between sampling and culturing specimens rarely exceeded 2 hours.

In both the elective grafts a transit isolator was used after a 'sterile' bowel had been achieved. The patients were transferred to a small transit isolator for 12 hours while the original isolator was emptied, resterilized, and restocked. Case 1, because of his hyperplastic marrow, received radiotherapy to areas of the iliac crests to create a 'space' for the new marrow. For this he was transferred by van to an associated hospital in the transit isolator and, on return, re-entered the tent, strict isolation having been maintained throughout. The 2 patients with SCID (Cases 3 and 4) did not receive immunosuppressive therapy before bone marrow grafting.

\section{Results}

During 15 months we have had 242 days of experience in treating patients-including one adult -in the two sizes of isolator. The Table summarizes clinical data, including the occurrence of leucopenia 


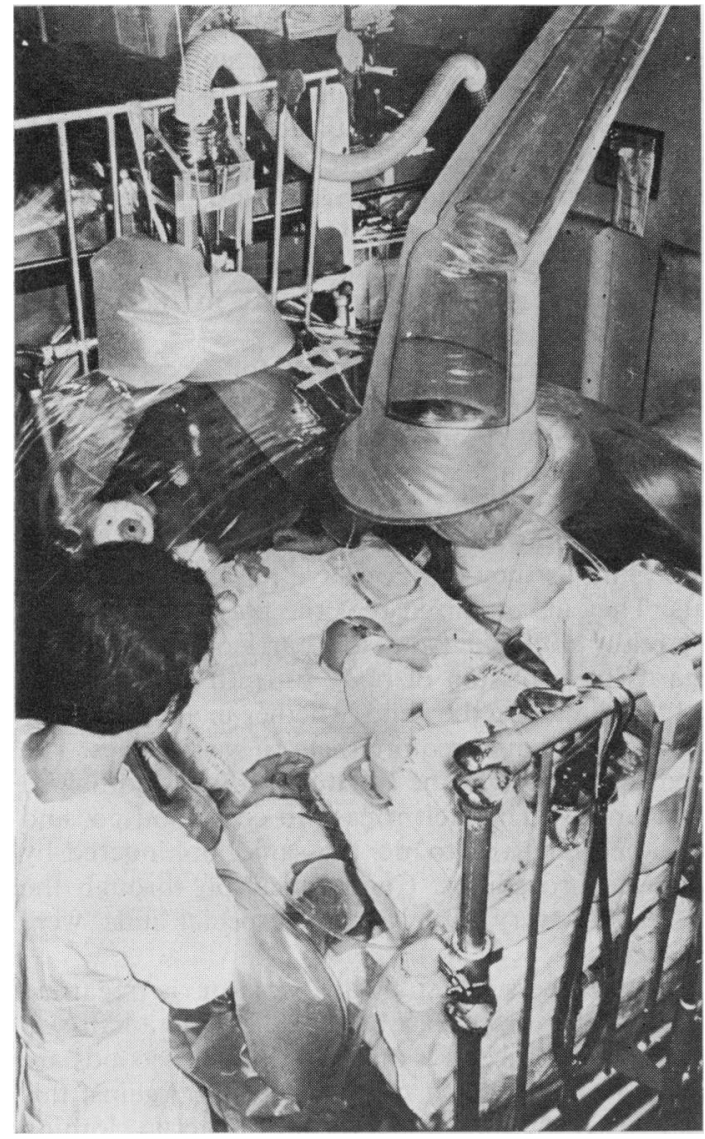

Fig. 2 General view of cot isolator in use.

and fever in the 4 children. The elective transplants (Cases 1,2) were approached cautiously in view of the high risks associated with vigorous immunosuppression. In Case 1, despite routine Hibiscrub and Naseptin at home, swabs from the nose and a sternal sinus yielded Staph. aureus in large numbers.
In addition, he had aspergillosis of the spine and had been pyrexial for several months before admission. CGD results in a hyperplastic bone marrow and the immunosuppression used while in the isolator reduced the neutrophil count to $<0.5 \times 10^{9} / 1$ $\left(500 / \mathrm{mm}^{3}\right)$ for 6 days. On 2 of these days the neutrophil count was $<0 \cdot 1 \times 10^{9} / 1$ with a total white cell count of $0.5 \times 10^{9} / 1\left(500 / \mathrm{mm}^{3}\right)$. Using the general measures outlined above, combined with clotrimazole and amphotericin B for the aspergillosis, no further infection occurred. However, Candida albicans appeared in the stools 10 days after the disappearance of bacteria. He remained in the tent for a total of 31 days.

Case 2 received a graft because of his increasing blood transfusion requirements, spontaneous bruising, and infective episodes. He had no unusual organisms on entry into the tent. His stools became sterile 4 days after full implementation of the decontamination procedures, and remained microbefree over the next 62 days. Though he was cooperative and capable of washing himself, decontamination was more effective when the nurses washed him. His white cell count stayed below $0.5 \times 10^{9} / 1\left(500 / \mathrm{mm}^{3}\right)$ for 14 days and his neutrophil count below $0 \cdot 1 \times 10^{9} / 1\left(100 / \mathrm{mm}^{3}\right)$ for 10 days and $<0.5 \times 10^{9} / 1\left(500 / \mathrm{mm}^{3}\right)$ for a further 11 days. Throughout his 72 days of isolation he remained free of infection.

The two infants (Cases 3,4), unlike the older children, required no immunosuppressive therapy and consequently neutropenia did not occur. The one patient (Case 3) who died of infection was heavily colonized by pathogens on admission. Pseudomonas aeruginosa was isolated from blood cultures and several other sites, and Salmonella typhimurium was isolated from stool cultures. Despite the decontamination regimen and ampicillin, carbenicillin, and gentamicin therapy the stools and blood cultures continued to yield salmonella and pseudomonas. A high fever, evident throughout the

Table Summary of clinical and haematological data on patients while in isolator

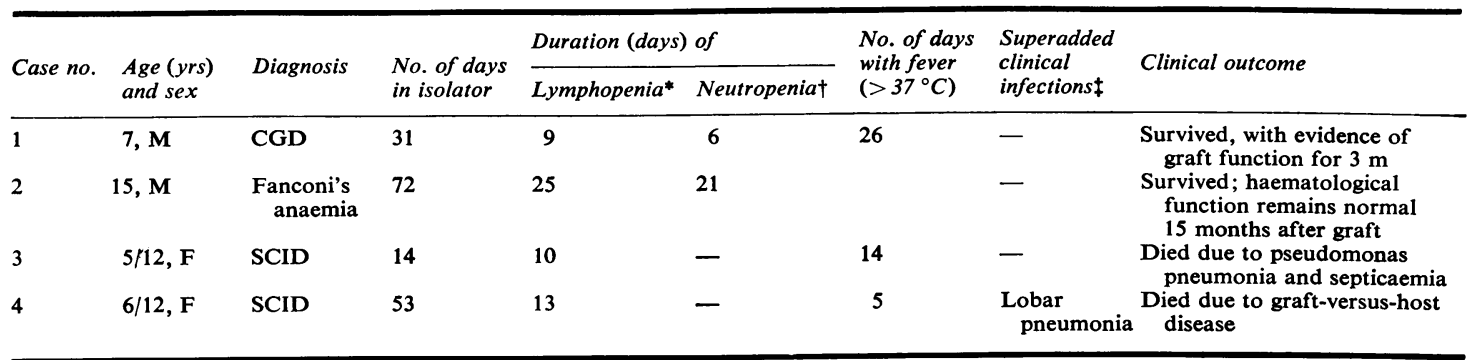

*Lymphocytes $<0.5 \times 10^{9} / 1$; †neutrophils $<0.5 \times 10^{9} / 1$; $\ddagger$ infections arising after entry into the isolator.

$\mathrm{CGD}=$ chronic granulomatous disease; SCID = severe combined immune deficiency disease. 
interval between admission and entry into the isolator, persisted. She died 14 days after isolation due to pseudomonas pneumonia and septicaemia.

Case 4 had been in the isolator for a total of 53 days during which time small numbers of Strep. pneumoniae and viridans streptococci were isolated from the oropharynx, and Staph. epidermidis from the skin, mouth, and vagina. All of these were present before entry. The second isolation of Strep. pneumoniae on the 13th day after entry into the tent coincided with the development of lobar pneumonia. Penicillin therapy led to rapid resolution of the pneumonia, and the pneumococcus was eliminated.

Case 4 died of myocarditis due to graft-versushost reaction 36 days after marrow transplantation. There was good evidence of immunological reconstitution. No viruses were identified by electron microscopy or isolated by culture, either before or after death and antibody studies were unhelpful.

In 3 of the patients, sterile stool cultures were achieved by the end of the first week of bowel decontamination. Subsequent stool cultures from these patients while in the isolator yielded no growth of organisms in $76 \%$ of the specimens. Stool cultures from the fourth patient, as already stated, continued to grow the pseudomonas and salmonella organisms present on admission.

The proportion of sterile cultures from the various other sites were as follows: axillae $92 \%$, hairline $85 \%$, groins $59 \%$, ears $68 \%$, nose $50 \%$, throat $71 \%$, mouth $50 \%$, urine $87 \%$, and vagina $91 \%$. The majority of the positive cultures consisted of Staph. epidermidis while scanty faecal organisms occurred in one-fifth of the specimens.

\section{Discussion}

Although tedious for patients and staff alike, strict protective isolation is a necessity in children with severe immunosuppression. Infection occurring in these patients after transplantation could have put impossible demands for granulocytes and platelets on the leucopheresis unit in addition to increasing the severity of any graft-versus-host disease (Thomas et al., 1975).

The acceptability of the Trexler form of isolator is not in doubt. The 2 children who were old enough to be fully aware of their surroundings accepted their terms of 31 and 72 days' isolation well. Case 1 regarded the isolator as a castle to keep germs at bay. Despite numerous games, school work, and even a ciné camera inside the isolator, boredom was a major problem. This was less so with Case 2 whose isolator was placed in an open ward and he was very much more part of the ward activities. It was some- times difficult to provide a sufficiently varied sterile diet but a small refrigerator in the supply compartment of the isolator provided useful cold drinks. Just how well the 2 infants accepted isolation is more difficult to assess. Case 4, judging by normal responses to the approach of staff and normal developmental progress while in the isolator, showed neither sensory nor emotional deprivation. Tests of vision and hearing while in the isolator gave normal results.

Parents also found the isolator acceptable in contrast to strict room isolation where they must either carry out rigorous aseptic rituals or be denied physical contact and close proximity. Though the plastic film did provide a physical barrier, parents were aware that they looked like parents to their child as they did not have to wear masks and hats. They also realized that the plastic barrier was not really different from the barrier of a gown as regards transmission of body warmth and contour, and that handling the child, whether in an isolator or in a cubicle, would require them to wear gloves. The parents were taught the isolator routines and helped considerably. They felt it easy to come and go, and for other relatives to do the same, unhindered by elaborate procedures. Communication through the plastic was very easy and no special aids were required.

For members of staff the isolator is regarded almost unanimously as a boon. Entry into a half-suit takes about 30 seconds and less than 15 seconds are needed to apply a pair of glove sleeves. Against this must be set the time taken to put on sterile clothing and the cost of numerous sets of these items. Some procedures do require novel adaptation, for example, auscultation with a stethoscope head passed down the sleeve into the gloved hand, but most procedures can be carried out normally (Fig. 3). The benefits of being able to approach and talk to the patient without formalities, such as gowning, are great, and allow much more contact between patient and staff than is usually possible during isolation.

With regard to prolonged isolation, the tent is greatly preferred to a cubicle by our nurses because, despite the need for some additional planning, the continuous maintenance of asepsis is easier and more certain.

Strict protective isolation has been shown to be superior to conventional room isolation in reducing the incidence of severe infections among adult leukaemia patients who were receiving intensive chemotherapy (Levine et al., 1973, 1975). Dooren and his colleagues (de Koning et al., 1969; Vossen et al., 1973) have used a laminar flow system for isolation of patients undergoing bone marrow transplantation. They achieved good results in 


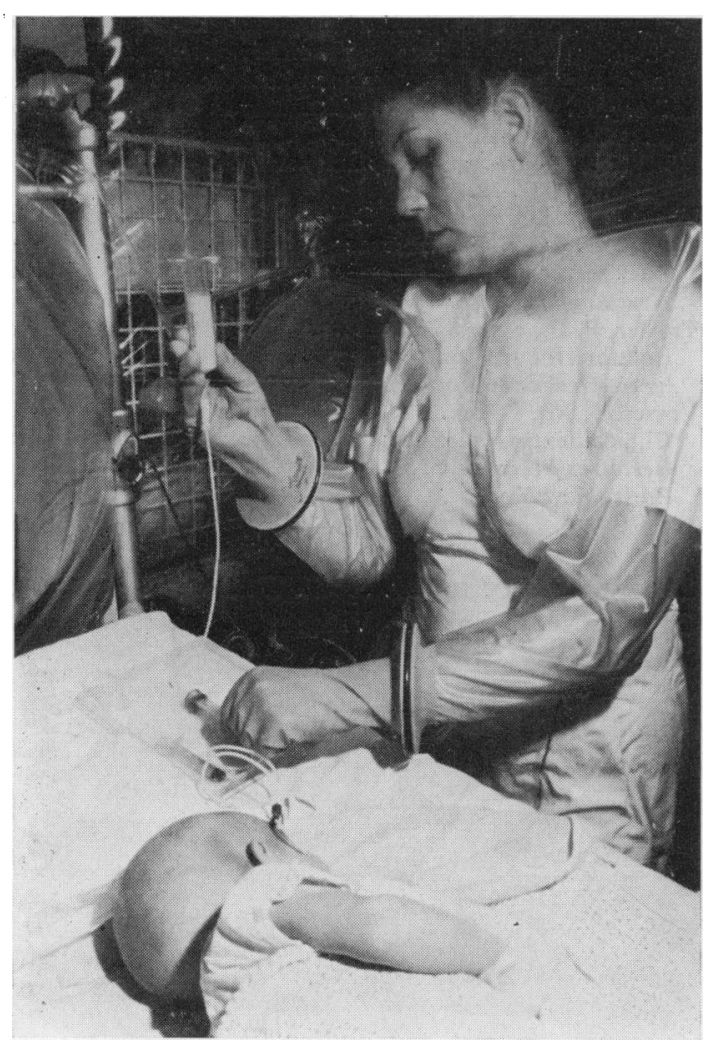

Fig. 3 Manipulation of nasogastric feed through glove sleeves in cot isolator.

preventing cross-infection over long periods of time. However, this form of isolation fails to provide a complete physical barrier between the patient and his environment and 2 of the 5 Leiden patients were colonized by exogenous micro-organisms.

In a recent comprehensive review (Thomas et al., 1975), infection is reported to be the 'usual proximate cause of death' during and after transplantation. The authors report that almost all of their marrow transplants were carried out using 'simple mask reverse isolation'. In an earlier series (Solberg et al., 1971), 41 infections occurred in 11 patients after transplantation. Infection with exogenous organisms was encountered most frequently in patients occupying conventional isolation rooms.

Except for the persistence of pathogenic bacteria in one patient, the decontamination procedures successfully suppressed to minimal levels the preexisting bacterial populations at the various sites. Recording, as we do, the most scanty cultures of commensal organisms tends to create a false impression of inadequacy in the decontamination procedures. However, we recognize the impossibility of maintaining true sterility of body surfaces that have previously been heavily colonized by commensals or potential pathogens. This is in contrast to the situation where babies are delivered aseptically and then maintained in completely germ-free conditions. But a full 'inventory' of even the most minimal numbers of organisms is necessary to forewarn against the hazard of subsequent overgrowth by such organisms.

The antibiotic combination used effectively suppressed the intestinal flora despite the fact that none of these agents individually is usually regarded as being active against the predominant Bacteroides group and other anaerobes of the bowel. This may be explained partly by the fact that these drugs achieve very high concentrations in the bowel from which they are not absorbed systemically and, in addition, an unsuitable milieu for anaerobes may result once the aerobic flora has been eradicated.

At the end of the period of decontamination and isolation patients can be discharged immediately from hospital to minimize the risks of recolonization by hospital pathogens. The alternative course which we have followed is to recolonize (or 'reconventionalize') the bowel with the donor's mixed flora after screening it for suitability.

An adult patient who recently underwent marrow transplantation at our hospital remained free of exogenous infection during 72 days' stay in the bed isolator despite a total white cell count of $<0 \cdot 1 \times 10^{9} / 1\left(100 / \mathrm{mm}^{3}\right)$ for 6 weeks. Throughout this time $C$. albicans was isolated from several mucosal sites and persisted despite systemic antifungal therapy. The patient eventually died of systemic candidiasis. This, and our additional experience with Case 1, has prompted us to administer nystatin or amphotericin B 4 days before colistin and framycetin in the decontamination of subsequent patients.

Because of its high acceptability from a microbiological, staff, and patient point of view, we recommend that this form of isolation be used more widely and thus undergo further evaluation.

We are grateful to Dr. K. Hugh-Jones for allowing us to report on his patients; to the nursing staff for their co-operation; to the technical staff of the Department of Bacteriology; and to the Andrew Bostic Fund for financial assistance.

\section{References}

de Koning, J., van Bekkum, D. W., Dicke, K. A., Dooren, L. J., van Rood, J. J., and Radl, J. (1969). Transplantation of bone-marrow cells and fetal thymus in an infant with lymphopenic immunological deficiency. Lancet, 1, 1223-1227. 
Gordon, H. A., and Pesti, L. (1971). The gnotobiotic animal as a tool in the study of the host microbial relationship. Bacteriological Reviews, 35, 390-429.

Humble, J. G., and Barrett, A. J. (1975). Technique of bone marrow transplantation. Proceedings of the Royal Society of Medicine, 68, 580-582.

Johnson, F. L., Hartmann, J. R., Thomas, E. D., Chard, R. L., Hersman, J. A., Buckner, C. D., Clift, R. A., and Storb, R. (1976). Marrow transplantation in treatment of children with aplastic anaemia or acute leukaemia. Archives of Disease in Childhood, 51, 403-410.

Levine, A. S., Siegel, S. E., Schreiber, A. D., Hauser, J., Preisler, H., Goldstein, I. M., Seidler, F., Simon, R., Perry, S., Bennett, J. E., and Henderson, E. S. (1973). Protected environments and prophylactic antibiotics. New England Journal of Medicine, 288, 477-483.

Levine, A. S., Robinson, R. A., and Hauser, J. M. (1975). Analysis of studies on protected environments and prophylactic antibiotics in adult acute leukaemia. European Journal of Cancer, 11, Suppl., 57-66.

Oh, M. H. K., Rodey, G. E., Good, R. A., Chilgren, R. A. and Quie, P. G. (1969). Defective candidacidal capacity of polymorphonuclear leukocytes in chronic granulomatous disease of childhood. Journal of Pediatrics, 75, 300-303.
Rodey, G. E., Park, B. H., Windhorst, D. B., and Good, R. A. (1969). Defective bactericidal activity of monocytes in fatal granulomatous disease. Blood, 33, 813-820.

Solberg, C. O., Meuwissen, H. J., Needham, R. N., Good, R. A., and Matsen, J. M. (1971). Infectious complications in bone marrow transplant patients. British Medical Journal, 1, 18-23.

Thomas, E. D., Storb, R., Clift, R. A., Fefer, A., Johnson, F. L., Neiman, P. E., Lerner, K. G., Glucksberg, H., and Buckner, C. D. (1975). Bone marrow transplantation. New England Journal of Medicine, 292, 832-843; 895-902.

Trexler, P. C., Spiers, A. S. D., and Gaya, H. (1975). Plastic isolators for treatment of acute leukaemia patients under 'germ-free' conditions. British Medical Journal, 4, 549-552.

Vossen, J. M., Dooren, L. J., and van der Waaij, D. (1973). Clinical experience with the control of the microflora. Germfree Research, p. 51. Ed. by J. B. Heneghan. Academic Press, New York.

Correspondence to Dr. J. G. Watson, Westminster Children's Hospital, Vincent Square, London SW1P 2NS. 